\title{
Important notice: Improving the quality of research reporting in NeuroRehabilitation
}

NeuroRehabilitation has joined a group of journals who recently committed to improve the consistency and quality of manuscripts submitted and published in the field of disability and rehabilitation. We strongly encourage authors to review and comply with the reporting guidelines relevant to their submissions. We are asking reviewers to evaluate submissions on the basis of their conformity to the guidelines. The table below provides information about guidelines for different study types.

\begin{tabular}{lll}
\hline Study Type & Name & Source \\
\hline Case reports & CARE & www.care-statement.org/ \\
Diagnostic accuracy & STARD & www.stard-statement.org/ \\
Observational studies & STROBE & http://strobe-statement.org/ \\
Randomized controlled trial & CONSORT & www.consort-statement.org \\
Systematic reviews, meta-analyses & PRISMA & www.prisma-statement.org/ \\
\hline
\end{tabular}

If you will be submitting a manuscript to NeuroRehabilitation, please take the opportunity to review the guidelines and organize your submission accordingly.

Over the next six months, we will be revising the authors' instructions and reviewers' criteria to be more consistent with the reporting guidelines. We hope that you share our enthusiasm in seeing implementation as an important opportunity to advance the field. Thank you for your consideration.

Jeffrey S. Kreutzer, Ph.D. Co-Editor-in-Chief

Nathan Zasler, M.D. Co-Editor-in-Chief 